\title{
Artificial Intelligence-Based Real-Time Signal Sample and Analysis of Multiperson Dragon Boat Race in Complex Networks
}

\author{
Yu Li and Peihua Liu \\ College of Physical Education, Beihua University, Jilin 132013, China \\ Correspondence should be addressed to Peihua Liu; liupeihua@beihua.edu.cn
}

Received 2 January 2022; Revised 24 January 2022; Accepted 26 January 2022; Published 23 February 2022

Academic Editor: Peican Zhu

Copyright (C) $2022 \mathrm{Yu} \mathrm{Li}$ and Peihua Liu. This is an open access article distributed under the Creative Commons Attribution License, which permits unrestricted use, distribution, and reproduction in any medium, provided the original work is properly cited.

\begin{abstract}
Dragon boat sport is a traditional activity in China. In recent years, dragon boat sport has become more and more popular around the world. In order to face more challenges, it is urgent for athletes to enhance their own strength. Scientific training methods are particularly important for athletes, and accurate training data are the basis to support scientific training. Traditional mathematical statistic methods neither can sample signals accurately nor can they do real-time analysis and feedback the characteristics to each athlete. In this paper, we use the wearable device with a triaxial accelerometer and heart rate sensor builtin to sample the speed signals and heart rate signals of athletes in various stages of men's $1000 \mathrm{~m}$ straight race. Based on the complex network theory, we regard the 23 dragon boat athletes in the dragon boat race as 23 nodes so as to establish a network with 23 nodes and reflect the importance of nodes by measuring the impact of node deletion on the results of the race. The neural network multilayer perceptron (MLP) model is used for training to obtain the optimal combined value with speed and heart rate for each race stage. The optimal value will be used in the simulated race as the target value to verify if it can help to improve the training efficiency. Experimental results show that the optimal value obtained by this method has a positive effect on the results of the dragon boat race which is beneficial to sports training and tactics planning.
\end{abstract}

\section{Introduction}

Dragon boat sport, commonly known as a dragon boat race, is an important part of traditional Chinese culture. In recent years, dragon boat sport began to be popular in many countries around the world, and dragon boat race spreads on all continents. Since 2010, the dragon boat race has been a part of the Asian Games. Chinese dragon boats are shown as a demonstration sport event during the canoeing competition at the Tokyo Olympic Games on August 3, 2021. Dragon boat as a demonstration sport event included in the Tokyo Olympic Games, which marked that dragon boat has started the Olympic application program. Promoting the dragon boat sports to the Olympic Games plays a very positive role in the external dissemination and display of Chinese culture; at the same time, enhancing the competitive ability has become the main direction in the development of the dragon boat sports [1].
In the 18th Asian Games in 2018, the Chinese dragon boat team got two gold medals and two silver medals in five individual events. The men's team and women's team both won gold medals in $200 \mathrm{~m}$ and silver medals in $500 \mathrm{~m}$. However, the men's $1000 \mathrm{~m}$ straight race failed [2]. According to the conclusion obtained from the research of paper $[2,3]$, for medium-short distance dragon boat races, such as $200 \mathrm{~m}$ and $500 \mathrm{~m}$, the tactical mode has relatively little influence on the results of the races, and the athletes basically rely on using all their strength. However, for long-distance dragon boat races, such as $1000 \mathrm{~m}$, although the result of the race has a lot to do with the speed ratio of each stage, the velocity structure is not only associated with tactical arrangements but also with the physical condition of athletes. It is known from paper [3] that the excellent start speed is important for victory, but the one-sided pursuit of high strength set sail will be easy to cause lactic acid accumulation so that athletes' overload movement affects the subsequent 
matches. Therefore, in addition to monitoring speed, athletes' physical condition monitoring is also particularly important, and heart rate is an important indicator to reflect athletes' physical condition. Only by adopting scientific training methods and carrying out targeted daily training under reasonable speed and heart rate, we can improve training efficiency and achieve better results in the longdistance dragon boat race.

The scientific training method depends on the sampling of athlete's physical data and motion data which can be analyzed in the process of the game. Effective data support is the basis for planning effective tactics. It is quite important to allocate speed reasonably at different stages of the competition according to the conditions of both sides. Therefore, real-time signal sample and analysis in the process of the dragon boat race is the key step to improve sports performance.

Traditional signal sampling and analysis methods can only be used for simple signals; it requires a lot of human intervention; moreover, they are time consuming and not accurate enough to complete real-time targeted signal sample and analysis. With the increasing development of artificial intelligence technology, wearable equipment technology is constant to promote the development of motion signal sampling. Relevant sensors such as acceleration and heart rate can be embedded in wearable devices such as sports bracelets to sample a large amount of signals in real time. At the same time, with the development of the artificial neural network, as we know from paper [4], multilayer perceptron (MLP) can be approximated to perform any function virtually to achieve the desired accuracy to fuse multiple features for optimal results. Thus, MLP is useful for us to research how to get the optimistic value in this paper. At the same time, it is also necessary to discuss the importance of each athlete and the impact of the association between athletes in the dragon boat race. The complex network theory in network science provides a theoretical basis for the discussion of the above issues [5,6]. The results also provide effective data support for the multilayer perceptron (MLP) model and make the optimistic value obtained more accurate.

Accordingly, the contributions of this paper are summarized as follows: (i) the real-time speed and heart rate signals of dragon boat athletes are sampled in each stage of the competition by wearable bracelets with triaxial accelerometer and heart rate sensors built-in and filtered separately; (ii)The MLP model is used to train the sampled signals, and the optimal combined value of heart rate and speed are combined; (iii) the optimal value feedback to coaches and athletes, the effectiveness of the optimal value is verified by experimental methods and evaluated whether it has a positive influence on training.

The rest of this paper is organized as follows. Section 2 reviews the related work. In section 3 , the proposed method is introduced. In section 4, the implementation method of signal sample in dragon boat sport is presented. Experimental design and results analysis are presented in sections 5 and 6, respectively. Section 7 concludes this paper and gives future research directions.

\section{Related Work}

Paper [7] was accomplished by monitoring dragon boat athletes who won the championship, and based on the research, the researchers found that there was a connection between the volume of training load intensity and motor function before the dragon boat competitions; the research showed that reasonable arrangements for the training content of each cycle, strength, and load had a significant impact on improving the capacity of the athletes' aerobic power and the athletes' performance. In [8], Parry examined dragon boat racing (DBR) for breast cancer survivors and revealed that DBR contributes to women's social, emotional, physical, spiritual, and mental health. In [9], Santiago and Sousa presented a survey on relevant work, current techniques, and trends in the area of team tracking systems applied to sports. Nikolakaki et al. showed that using final score difference provides yet a better prediction metric for competitive balance in 2020 [10]. Wei et al. focused on the historical evolution of the dragon boat race and the development of sports nonmaterial cultural heritage in [11].

Paper [12] discussed that the wearable input device could provide the user with one data input device and authentication system that was portable and could be worn like a fashion accessory. Paper [13] surveyed the state of the art in HAR based on wearable sensors and also proposed a twolevel taxonomy in accordance with the learning approach (either supervised or semisupervised) and the response time (either offline or online). In [14], Wang et al. introduced the state-of-art sensor modalities in HAR and talked more about the techniques involved in each step of wearable sensor modality-centred HAR in terms of sensors, activities, data preprocessing, feature learning, and classification, including both conventional approaches and deep learning methods. In paper [15], Montalto exploited a wearable device equipped with inertial sensors such as accelerometer, gyroscope, and compass used for behavioral analysis (BA) focused on human activity recognition (HAR) to evaluate quantity and quality of movements. Paper [16] exploited MuSA (a tiny device, suitable for being worn at the belt and embedding an Inertial Measurement Unit) to enable useraware behavioral analysis based on environmental devices. Paper [17] used wearable sensor arrays for multiplexed insitu perspiration analysis. Paper [18] discussed the type of methods and technologies used for HAR, IL, and data fusion, and the precision obtained for them were performed. Paper [19] provided a review of recent trends in the area of wearable sweat sensing with discussions on relevant topics of interest in material science, device development, sensing mechanisms, power generation, and data management.

In [20], Assah et al. examined the validity of a combined heart rate and motion sensor in estimating physical activity energy expenditure (PAEE) in free-living adults. From [21], we know that the heart rate was a very sensitive physiological parameter to reflect the metabolic rate and played an important role in scientific training. In paper [22], XBee wireless sensor networks for heart rate monitoring are used as a training aid for various types of sports. The paper [23] described the development of a triaxial accelerometer and a 
portable data processing unit for the recognition of daily activity. Reference [24] introduced a generic framework for the automated classification of human movements using an accelerometry monitoring system. The framework was structured around a binary decision tree in which movements were divided into classes and subclasses at different hierarchical levels, and a better effect was achieved.

In [25], Naughton researched on artificial intelligence (AI) and training in 1987. Ennals performed practical applications of AI in education and training in 1990 in [26]. Paper [27] undertook a short review of scientific research studies centered on the contemporary development of AI and probabilities of its applied use in sports practice. Paper [28] examined the possibilities promised for the sports environment by new technologies such as big data and AI. The technologies' capacity for a more precise data sample and analysis could enhance sports-related performance.

Neural networks were used increasingly as statistical models. In [29], the multilayer perceptron as an approximation to a Bayes optimal discriminant function was proposed in a paper in 1990. In paper [30] the analysis of the relationship between the hidden nodes and the fault tolerance of the multilayer perceptron was given. Paper [4] was dedicated to the analysis of a limited training dataset influence on the performance of MLP as a nonparametric pattern classifier. Paper [31] presented the performance comparison between multilayer perceptron (back propagation, delta rule, and perceptron). In paper [32], an extended backpropagation algorithm that allowed all elements of the Hessian matrix to be evaluated exactly for a feedforward network of arbitrary topology was present in 2014. In [33], the findings of the research showed that artificial neural networks could be used for parameter estimation in cause-effect based studies. In [34], the performance of MLP and that of linear regression (LR) were compared, with regard to the quality of prediction and estimation and the robustness to deviations from underlying assumptions of normality and independence of errors. In [35], the authors proposed a new neural network model called multilayer perceptron with embedded feature selection (MLP-EFS). In [36], the authors proposed a selfish group optimization algorithm (OISHO) based on orthogonal design and information update. In [37], the authors proposed an improved whale optimization algorithm based on teaching and learning based on the simplex method (TSWOA).

\section{The Proposed Method}

The overall process of sampling the speed signals and heart rate signals of dragon boat athletes in various stages of training or competition based on wearable devices is shown in Figure 1. Firstly, human acceleration and heart rate signals are sampled by wearable devices with builtin triaxial acceleration and heart rate sensors, and the acceleration and heart rate signals are filtered and preprocessed, respectively. Based on the limit of sample amount, in this paper, the optimal value is estimated by the MLP model based on the dataset composed of dragon boat race data and simulated training race data.
3.1. Human Motion Model. Under normal circumstances, if the movement does not involve the rotation of the human body, the human body can be simplified as a multiparticle model. When studying body movement, the body can be regarded as a particle. However, the dragon boat sport is a compound motion, which has both translation and rotation in the process of movement so that the shape and size of the human body need to be considered [38]. Therefore, in the dragon boat sport, we regard the human body as a multirigid body system, and the rigid body is a continuous point composed of a series of particles with a constant distance from each other.

Havana is widely used at present, as shown in Figure 2, which divides the human body into 15 segments, each of which is connected by ball hinges.

3.2. Wearable Device. A wearable device is a portable device that can be worn directly on the body and worn on the hand or integrated into the user's clothing or accessories. It is not only a hardware device but also can achieve powerful functions through software support, data interaction, and cloud interaction [39]. With the development of artificial intelligence technology, sensors have been built into various mobile devices, featuring low power consumption, easy portability, accurate data, and no limitation with scenarios. Wearable devices can be used to record daily life, physical health, etc., and can also be used in sports analysis.

Many types of sensors that can be built in wearable devices, Zigbee, WiFi, Bluetooth, etc., are the common way of wireless communication. Concrete implementation mode is to built the sensors through the embedded technology in wearable devices. The physiological signals will be mapped to the activities of athletes after getting enough signals by monitoring athlete's training process effectively. The whole behavior can be used for the condition of athletes training, to help assistant coach establish more scientific and more targeted training plan.

3.3. Multilayer Perceptron. Multilayer perceptron (MLP) is an artificial neural network, including input layer, multihidden layer, and output layer. The neural network of multilayer perceptron is shown in Figure 3. If the perceptron contains only one hidden layer, MLP contains $n$ neurons, indicating input $\mathrm{n}$-dimensional signals, and $m$ output neurons, indicating include $\mathrm{m}$ types of output. The more hidden layers and the more hidden neurons, the better fitting ability of the model. Perceptron neural network is defined as follows:

$$
\begin{aligned}
& u=\sum_{i=1, j=1}^{n, m} w_{i j} x_{i}+b, \\
& y=\operatorname{sign}(u)= \begin{cases}+1, & u<0, \\
-1, & u \geq 0,\end{cases}
\end{aligned}
$$

where $b$ is the bias in the MLP model.

In order to minimize the loss function value, the model is trained by the reverse gradient propagation algorithm. Back 


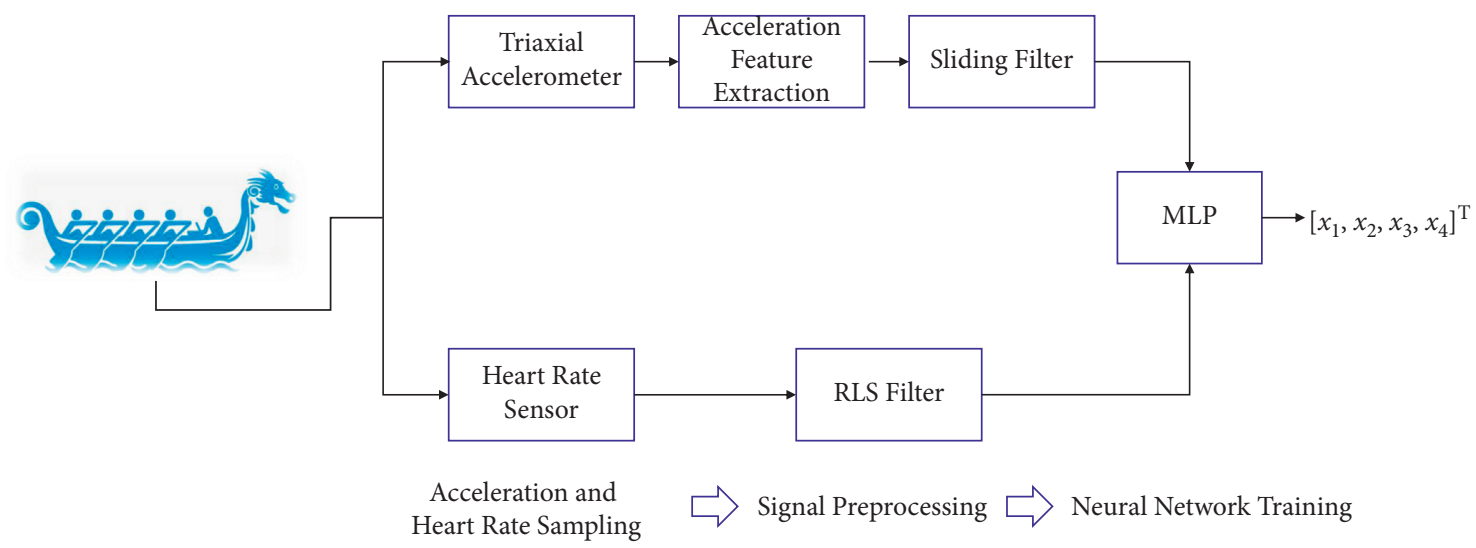

FIgURE 1: Overall process.

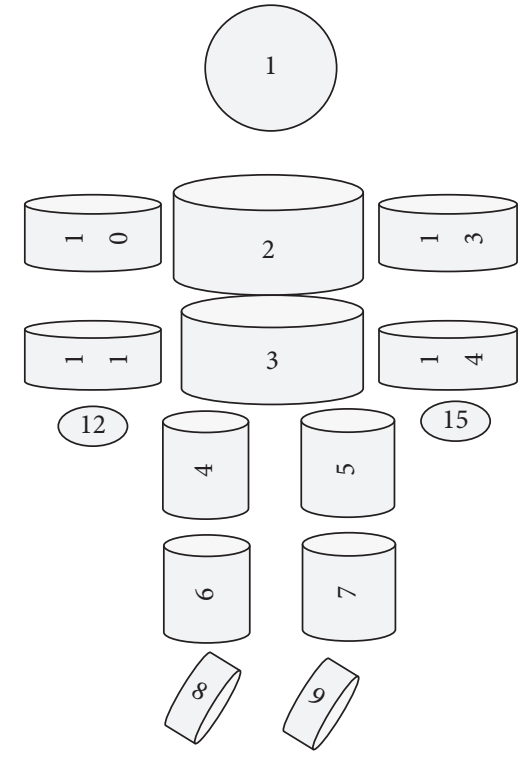

Figure 2: Human model.

propagation is used for weight updates to bring the network output closer to the label.

\section{Implementation Method of Signal Sample in Dragon Boat Sport}

As the issue of identifying athletes' speed and heart rate at each race stage, it is essentially a process of sampling athletes' body signals through sensors and using the processing unit in wearable devices for signal processing, and the design of signal sample scheme is one of the key contents for this paper. Whether the design of the signal sample scheme is effective or not will affect the reliability of the signal sample directly and affect the training results at the same time.

4.1. Design Scheme of Signal Sample. Dragon boat sport is mainly the upper limb sport, so this paper is mainly designed to comprehensively sample the signals of the upper limb movement during boating.
In order to obtain the acceleration signals of the upper limb movement in the rowing process through understanding the human body model, the acceleration signals of the arm in the three-axis direction are needed. In the process of the signal sample, a device with a three-axis acceleration sensor and a heart rate sensor is worn on the wrist of the athlete, and the acceleration signals of each axis and the heart rate signals of the athlete are sampled during boating.

4.2. Feature Value Extraction Based on Acceleration Signal. As shown in Figure 4, generally, the human body will generate acceleration components in three directions in the process of movement: forward, lateral, and vertical direction so as to build the motion model of the human body. Since dragon boat sport also involves forward, lateral, and vertical behaviors, we have built a three-axis acceleration sensor into the wearable sports bracelet, which can detect the movement speed more accurately.

Ideally, the three directions are exactly corresponding to the three axes, but in practice, the angle of the three axes of the sensor device is unknown, and the measured value of the three axes is the projection of the motion acceleration on the axis. Since rowing action is regular, we regard one swing as a unit movement cycle. In every time swing, the forward $X$, lateral $\mathrm{Y}$, and vertical $Z$ acceleration will have periodic changes, and the speed and acceleration sampled by the sensor will change all the time. In order to make the sample simpler and eliminate coordinate mapping of acceleration output values at different moments, we adopt the vector sum of triaxial acceleration as characteristic values of the motion state of the rotor, denoted as St, where $\alpha_{x, t}, \alpha_{y, t}$, and $\alpha_{z, t}$ are the acceleration values obtained by the acceleration sensor at time $t$ in $x, y$, and $z$ three-dimensional space, respectively; then,

$$
S_{t}=\sqrt{\alpha_{x, t}^{2}+\alpha_{y, t}^{2}+\alpha_{z, t}^{2}}
$$

4.3. Acceleration Signal Filtering. As the sampled triaxial acceleration signal contains a lot of interference signals and noises, in order to facilitate the later processing of the signal, 


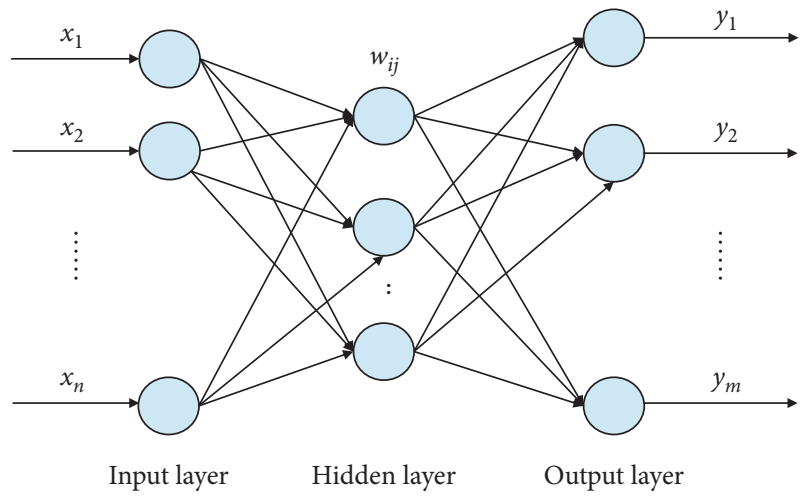

Figure 3: MLP model.

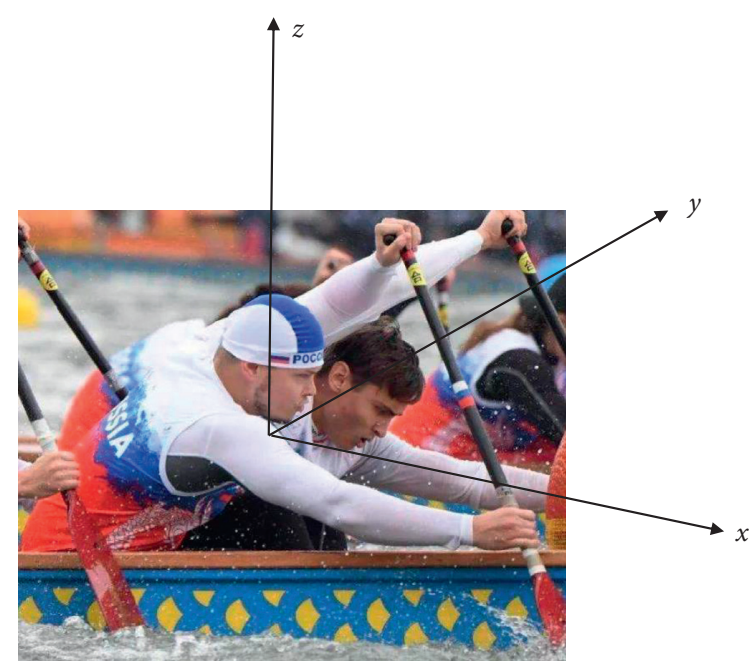

Figure 4: Movement direction.

the signal needs to be filtered first. The sliding filtering algorithm is better than the average filtering algorithm in real time and filtering effect so that the filtered waveform is smoother. So, this paper chooses the sliding filtering algorithm. Specific methods are as follows:

The system first sets the sampling interval as $d_{t}$ and a cache that can store four kinds of Data $\left(\right.$ Data $\left._{0} \sim \mathrm{Data}_{3}\right)$ and initializes the Data buffer to 0. The buffer Data is moved to the right, the original rightmost Data Data 3 is discarded, and the new sampled Data is moved to the position Data ${ }_{0}$. Then, the cache Data is averaged once, and the filtered value is obtained before the next sampling.

\subsection{Extraction of Characteristic Values of Heart Rate Signals.} In this paper, the wearable bracelet with a builtin heart rate sensor is selected to sample the heart rate signal because the heart rate signal sampled by wearable devices is often mixed with different degrees of exercise interference due to human activities, the frequency of interference signal overlaps with that of heart rate signal, and the signal is distorted and deformed, thus affecting the accuracy of heart rate detection. Therefore, the sampled signals need to be filtered first.
Considering the randomness of interference, in order to improve the accuracy of extracting heart rate signal, this paper adopts an RLS adaptive filter to denoise the heart rate signal combined with the acceleration signal. It not only considers the adaptive adjustment of filter parameters with the change of external interference but is also easy to do realtime calculation in wearable devices. By analyzing the characteristics of acceleration and the correlation degree of motion, an axial acceleration signal was selected as the expected signal, and the heart rate signal after preprocessing was filtered [40]. Then, the heart rate signal is denoised with the acceleration signals of the other two axes, and a better effect can be obtained.

It is assumed that the acceleration signal is used to simulate the motion interference, and the acceleration signal is used as the expected input signal in the adaptive filter to remove the motion interference. The signal model is expressed in formula 4 as follows, where $\mathrm{HRS}_{\text {in }}$ represents the heart rate signal under interference, $\mathrm{HRS}_{\text {out }}$ represents the filtered signal, $\alpha_{x}, \alpha_{y}$, and $\alpha_{z}$ represent the acceleration signal of three axes, respectively, $\lambda$ is the weight of HRS $_{\text {out }}$ in $\mathrm{HRS}_{\text {in }}, w_{x}, w_{y}$, and $w_{z}$ represent the weight of the triaxial acceleration signal, respectively.

$$
\begin{aligned}
\mathrm{HRS}_{\text {in }(i)}= & \lambda \times \mathrm{HRS}_{\text {out }(i)}+\alpha_{x(i)} \times w_{x}+\alpha_{y(i)} \\
& \times w_{y}+\alpha_{z(i)} \times w_{z} .
\end{aligned}
$$

In the process of motion, a signal model with motion interference is constructed for different coaxial acceleration signals, as shown in the following formula:

$$
\begin{aligned}
& \mathrm{HRS}_{i n(i)}=a_{1} \times \mathrm{HRS}_{\text {out }(i)}+b_{1} \times \alpha_{x(i)}, \\
& \mathrm{HRS}_{\text {in }(i)}=a_{2} \times \mathrm{HRS}_{\text {out }(i)}+b_{2} \times \alpha_{x(i)}+c_{1} \times \alpha_{y(i)}, \\
& \mathrm{HRS}_{\text {in }(i)}=a_{3} \times \mathrm{HRS}_{\text {out }(i)}+b_{3} \times \alpha_{x(i)}+c_{2} \times \alpha_{z(i)} .
\end{aligned}
$$

Three denoised heart rate signals can be obtained by the RLS adaptive filter. The original heart rate signal was taken as the input, and the acceleration signal of the three axes was successively taken as the reference input signal to get the denoised output heart rate signal.

\section{Experimental Design}

\subsection{Evaluation of the Optimal Value Based on the MLP Model}

5.1.1. Experimental Dataset Sources. Assuming that every $250 \mathrm{~m}$ in the men's $1000 \mathrm{~m}$ straight race is a stage, each race had four stages. 500 groups of sample data which included heart rate and speed signals are used to train the combined optimal value in this paper by $23 \%$ from each actual men's $1000 \mathrm{~m}$ straight race, $77 \%$ were derived from the men's $1000 \mathrm{~m}$ straight simulation race, samples of each group were composed of six dragon boat teams, due to the particularity of the dragon boat sport, and the signal sample was more difficult than other daily sports, Therefore, this paper adopts an artificial feature extraction method to extract feature values [41], and the extracted feature values which have fourstage data are used as the input dataset of the MLP model. 
TABLE 1: Score ranking from first 3 races.

\begin{tabular}{lccccc}
\hline Team & Race 1 point & Race 2 point & Race 3 point & Total score & Ranking \\
\hline A & 6 & 6 & 6 & 18 & 13 \\
B & 5 & 4 & 4 & 10 & 2 \\
C & 2 & 3 & 5 & 9 & 3 \\
D & 4 & 2 & 3 & 4 & 4 \\
E & 1 & 1 & 1 & 5 & 4 \\
F & 3 & 5 & 1 & 4 \\
\hline
\end{tabular}

TABLe 2: Score ranking from next 3 races.

\begin{tabular}{lccccc}
\hline Team & Race 4 point & Race 5 point & Race 6 point & Total score & Ranking \\
\hline A & 4 & 4 & 4 & 12 & 18 \\
B & 6 & 6 & 6 & 8 & 1 \\
C & 3 & 3 & 2 & 10 & 4 \\
D & 5 & 2 & 3 & 5 & 3 \\
E & 2 & 1 & 1 & 4 & 7 \\
F & 1 & 5 & & 5 \\
\hline
\end{tabular}

5.1.2. MLP Model. In this paper, a 7-layer perceptron was used to train the samples, semisupervised by real race results. 500 groups of sample data of the dragon boat race were input, consisting of two-dimensional features of speed and heart rates. We also regarded 23 athletes in the dragon boat race as 23 nodes so as to establish a network with 23 nodes and imported the result which reflects the importance of nodes by measuring the impact of node deletion on the results of the race as one of the MLP input. Loss function loss is $\mathrm{L} 2$, the learning rate is 0.01 , and training algebra epoch $=100$.

The above is to evaluate the combined optimal value of speed and heart rate at each stage which has a positive impact on grades.

5.2. Validity Verification of the Optimal Value. All dragon boat athletes who were participating in the experiment wore hand bracelets with a triaxial accelerometer and heart rate sensor. We used 6 same teams, called team A, B, C, D, E, and F, to simulate 6 groups of men's $1000 \mathrm{~m}$ straight dragon boat race. For the first 3 races, team $A$ would execute the optimal strategy; for the next 3 races, team B would execute the optimal strategy, and other teams would play freely; ifa team won the first prize in each race, it would get 6 points, in descending order, and the last one would only get 1 point. When the competition is finished, the total score and ranking of each team in the first 3 races and the last 3 races would be counted, respectively.

\section{Experimental Results and Analysis}

6.1. Score Ranking Results and Analysis. The experimental results of the simulated men's $1000 \mathrm{~m}$ straight races are shown in the following Tables 1 and 2 .

Through the above experimental results, we could observe that A team got the highest score in the first three men's $1000 \mathrm{~m}$ dragon boat races and B team got the highest score in the next three men's $1000 \mathrm{~m}$ dragon boat races.
This result shows that

(i) It is feasible to sample speed and heart rate signals by means of a builtin triaxial accelerator and heart rate sensor in wearable devices

(ii) The strategy formulation based on the speed ratio and the physical condition of the athletes had a great influence on the results of long-distance dragon boat race. The importance of each athlete and the impact of the association between athletes in the dragon boat race also had influence on the optimal values obtained.

(iii) The optimal values which was trained by the MLP model have positive influence to $1000 \mathrm{~m}$ straight dragon boat race, not only can sample speed and heart rate signals in real time but also can assist the coach to make reasonable competition tactics.

6.2. Analysis Time. Analysis time is an important metric in MLP model construction. In this study, MLPs-EFS [35], OISHO [36], TSWOA [37], and the proposed method were used for comparison. As shown in Figure 5, the analysis time of the algorithm proposed in this paper was the shortest. After the 700th iteration, the analysis time tended to be stable and always below $100 \mathrm{~ms}$. In contrast to the other three baselines, the analysis time of MLP-EFS and OISHO showed an exponential growth trend with the increasing number of iterations. This also showed the stability and flexibility of the algorithm proposed in this paper.

\section{Future Works and Prospects}

In this paper, the speed and heart rate signals of dragon boat athletes in the race are obtained through wearable devices. After effective processing, they are input into the MLP model as samples for training, and the optimal values of speed and heart rate at each stage of the dragon boat race are obtained, then used in the men's $1000 \mathrm{~m}$ straight simulation race. The optimal value strategy is tested, and the experimental results 


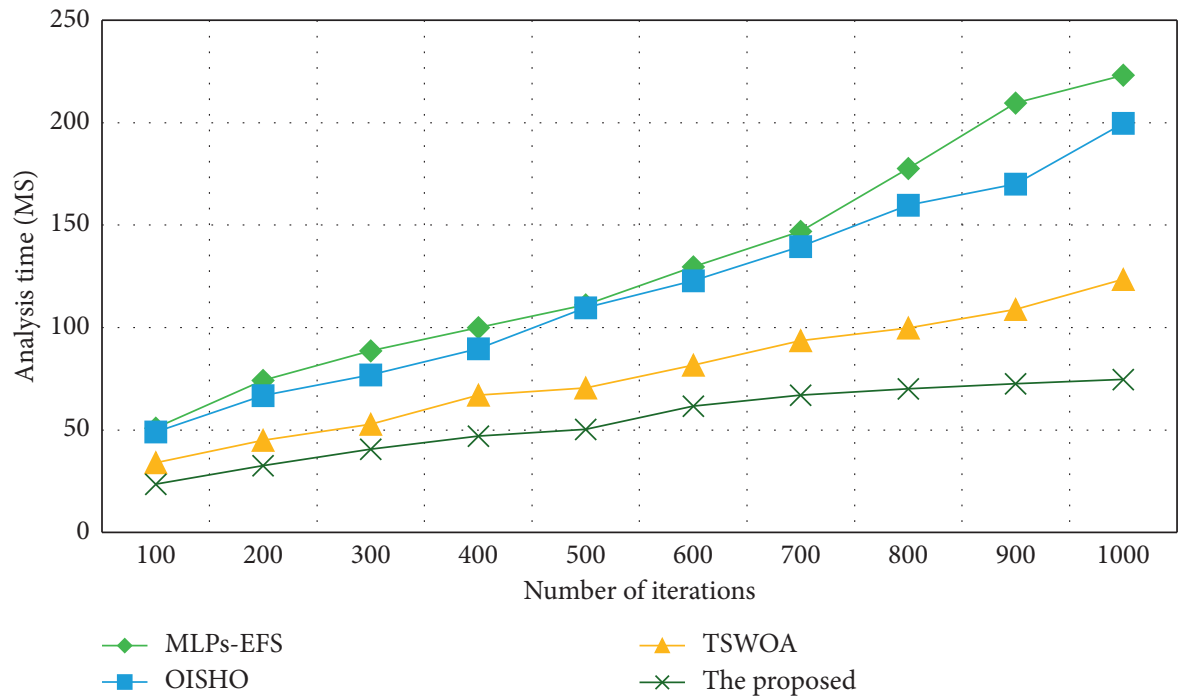

FIGURE 5: Results of analysis time.

prove that this value has a positive effect on the performance and tactics of the dragon boat sport. Due to the complexity of the dragon boat sport, the physiological requirements for athletes are relatively strict. In future research, we will not only improve the accuracy but also add more related physiological characteristics such as blood oxygen to extract more multidimensional optimal values to assist the coach in the training process. More effective dragon boat race tactics are formulated.

The number of layers of hidden layers of multilayer perceptron proposed in this paper has not been determined. With the rapid development of the deep neural network, designing a more complex network model should be able to better obtain the optimal value of speed and heart rate in each stage of the dragon boat race so as to improve the accuracy of the simulation.

\section{Data Availability}

All data used to support the findings of the study are included within this paper.

\section{Conflicts of Interest}

The authors declare that there are no conflicts of interest.

\section{Acknowledgments}

This work was supported by Dragon Boat Special Project in Beihua University in 2018 (Grant No. 2018LZ001) and Key Teaching and Research Projects in Beihua University in 2021 (Grant No. XJZD2021028).

\section{References}

[1] L. Bing and Z. Zhang, The Establishment of critical Power Model of Dragon Boat Athlete and Practical Research, IEEE, Mahattan, NJ, USA, 2011.
[2] S. L. Xu, "Kinematics analysis of the sitting position paddling technique of Chinese university student men's dragon boat $500 \mathrm{~m}$ straight race," China Sport Science and Technology, vol. 31, pp. 78-81, 2021.

[3] Z. Z. Yu, S. Liu, and J. J. Tan, "Speed structure analysis of men's $1000 \mathrm{~m}$ straight dragon boat race in the 18th," Asian Games, vol. 2021, no. -16, pp. 220-221, 2019.

[4] M. Z. Markovic, M. M. Milosavljevic, and A. B. Samcovic, "A Performance Analysis of the Multilayer Perceptron in Limited Training Data Set conditions," in Proceedings of the International Conference on Digital Signal Processing, July 1997.

[5] L. Zhang, J. Cao, and J. Li, "Complex networks: Statistical properties, community structure, and evolution," Mathematical Problems in Engineering, vol. 2015, Article ID 590794, 8 pages, 2015.

[6] Q. Sun, G. Yang, and A. Zhou, "An entropy-based selfadaptive node importance evaluation method for complex networks," Complexity, vol. 2020, Article ID 4529429, 13 pages, 2020.

[7] M. Y Xiao and Z. Zhang, "The controlling research on physiological index and biochemical index in major cycle of men dragon-boat athletes," in Proceedings 2011 International Conference on Human Health and Biomedical Engineering, August 2011.

[8] D. C. Parry, "The contribution of dragon boat racing to women's health and breast cancer survivorship," Qualitative Health Research, vol. 18, no. 2, pp. 222-233, 2008.

[9] C. B. Santiago and M. L. Sousa A Estriga, "Survey on team tracking techniques applied to sports," in Proceedings of the International Conference on Autonomous \& Intelligent Systems, May 2010.

[10] S. M. Nikolakaki, O. Dibie, and A. Beirami, "Competitive Balance in Team Sports Games," 2020, https://arxiv.org/abs/ 2006.13763

[11] T. S. Wei, W. R. Huang, and T. T. Mao, The Historical Evolution of Jimei Dragon Boat Race and Enlightenment to the Folk Sports Heritage, Fujian Sports Science and Technology, Fuzhou, China, 2014.

[12] J Ng, "Universal," 2009. US, US20090146947 A1.

[13] L. D. Oscar and Labrador, "A survey on human activityu recognition using wearable sensors," IEEE Communications Surveys \& Tutorials, vol. 15, no. 3, pp. 1192-1209, 2013. 
[14] Y. Wang, S. Cang, and H. Yu, "A Survey on wearable sensor modality centred human activity recognition in health care," Expert Systems with Applications, vol. 15, 2019.

[15] F. Montalto, C. Guerra, and V. Bianchi, MuSA: Wearable Multi Sensor Assistant for Human Activity Recognition and Indoor Localization, Springer International Publishing, New York, NY, USA, 2015.

[16] V. Bianchi, C. Guerra, and I. D. Munari, "Wearable sensors for behavioral assessment," Gerontechnology, vol. 15, p. 5, 2016.

[17] W. Gao, S. Emaminejad, H. Y. Y. Nyein et al., "Fully integrated wearable sensor arrays for multiplexed in situ perspiration analysis," Nature, vol. 529, no. 7587, pp. 509-514, 2016.

[18] J. Cerón and D. M. López, "Human activity recognition supported on indoor localization: a systematic review," Studies in Health Technology and Informatics, vol. 249, pp. 93-101, 2018.

[19] C. Legner, U. Kalwa, and V. Patel, "Sweat sensing in the smart wearables era: towards integrative, multifunctional and bodycompliant perspiration analysis," Sensors and Actuators A Physical, vol. 249, p. 296, 2019.

[20] F. K. Assah, U. Ekelund, S. Brage, A. Wright, J. C. Mbanya, and N. J. Wareham, "Accuracy and validity of a combined heart rate and motion sensor for the measurement of freeliving physical activity energy expenditure in adults in Cameroon," International Journal of Epidemiology, vol. 40, no. 1, pp. 112-120, 2011.

[21] R. C. Xmhao, "Real-time Heart Rate Monitoring in Sport Training," Journal of Chengdu Physical Education Institute, vol. 40, 2002.

[22] N. Zulkifli, F. Harun, and N. S. Azahar, "XBee wireless sensor networks for heart rate monitoring in sport training," Applied Physics A, vol. 106, no. 2, pp. 295-307, 2012.

[23] C. V. C. Bouten, K. T. M. Koekkoek, M. Verduin, R. Kodde, and J. D. Janssen, "A triaxial accelerometer and portable data processing unit for the assessment of daily physical activity," IEEE Transactions on Biomedical Engineering, vol. 44, no. 3, pp. 136-147, 1997.

[24] M. J. Mathie, B. G. Celler, N. H. Lovell, and A. C. F. Coster, "Classification of basic daily movements using a triaxial accelerometer," Medical, \& Biological Engineering \& Computing, vol. 42, no. 5, pp. 679-687, 2004.

[25] J. Naughton, "Artificial intelligence and education and training,” Artificial Intelligence, vol. 1, no. 3, pp. 77-82, 1987.

[26] R. Ennals, "Practical applications of artificial intelligence in Education and Training," Artificial Intelligence, Culture \& Language: on Education \& Work, vol. 1, 1990.

[27] M. Shestakov, "Artificial intelligence in sport science of the 21st century," Theory \& Practice of Physical Education, vol. 7, pp. 8-14, 2000.

[28] B. Torgler, Big Data, Artificial Intelligence, and Quantum Computing in Sports, Springer, New York, NY, USA, 2020.

[29] D. W. Ruck, S. K. Rogers, M. Kabrisky, M. E. Oxley, and B. W. Suter, "The multilayer perceptron as an approximation to a Bayes optimal discriminant function," IEEE Transactions on Neural Networks, vol. 1, no. 4, pp. 296-298, 1990.

[30] I.-C. Jou, S.-S. You, and L.-W. Chang, "Analysis of hidden nodes for multi-layer perceptron neural networks," Pattern Recognition, vol. 27, no. 6, pp. 859-864, 1994.

[31] M. K. Alsmadi, K. B. Omar, and S. A. Noah, "Performance comparison of multi-layer perceptron (Back propagation, Delta Rule and perceptron) algorithms in Neural Networks," in Proceedings of the IEEE International Advance Computing Conference, March 2009.

[32] C. Bishop, "Exact calculation of the hessian matrix for the multilayer perceptron," Neural Computation, vol. 4, no. 4, pp. 494-501, 2014.

[33] M. Kayri and O. Cokluk, "Data optimization with multilayer perceptron neural network and using new pattern in decision tree Comparatively," Journal of Computer Science, vol. 6, no. 5, pp. 606-612, 2010.

[34] J. Gaudart, B. Giusiano, and L. Huiart, "Comparison of the performance of multi-layer perceptron and linear regression for epidemiological data," Computational Statistics \& Data Analysis, vol. 44, no. 4, pp. 547-570, 2018.

[35] L. Bo, W. Ling, and L. Jiao, "Multi-layer perceptrons with embedded feature selection with application in cancer classification," Chinese Journal of Electronics, vol. 15, no. 4A, pp. 832-835, 2006.

[36] R. Zhao, Y. Wang, and P. Hu, "Selfish herds optimization algorithm with orthogonal design and information update for training multi-layer perceptron neural network," Applied Intelligence, vol. 49, no. 6, pp. 2339-2381, 2016.

[37] Y. Zhou, Y. Niu, Y. Niu, Q. Luo, and M. Jiang, "Teaching learning-based whale optimization algorithm for multi-layer perceptron neural network training," Mathematical Biosciences and, vol. 17, no. 5, pp. 5987-6025, 2020.

[38] J. Hu, J. Chen, P. Zhu, S. Huo, and M. Wang, "Difference and cluster analysis on the carbon dioxide emissions in China during COVID-19 lockdown via a complex network model," Frontiers in Psychology, vol. 12, 2022.

[39] J. Hu, C. Xia, H. Li, P. Zhu, and W. Xiong, "Properties and structural analyses of USA's regional electricity market: a visibility graph network approach," Applied Mathematics and Computation, vol. 385, Article ID 125434, 2020.

[40] J. Lv, X. Wang, K. Ren, M. Huang, and K. Li, "ACO-inspired Information-Centric Networking routing mechanism," Computer Networks, vol. 126, pp. 200-217, 2017.

[41] L. Ma, M. Huang, S. Yang, R. Wang, and X. Wang, "An adaptive Localized decision variable analysis approach to large-scale multiobjective and many-objective optimization," IEEE Transactions on Cybernetics, 2021. 